\title{
Italian research \\ on the Antarctic atmosphere
}

\author{
Michele Colacino and Claudio Rafanelli \\ Istituto di Scienze dell'Atmosfera e del Clima (ISAC), CNR, Roma, Italy
}

\begin{abstract}
In this work after a short introduction on the structure of Programma Nazionale di Ricerche in Antartide, the different researches on atmospheric physics developed by Italian scientists are presented. The international activities are described, with particular attention both to APE experiment and the setting up of the new base in Dome-C. The results obtained are also discussed and the new projects proposed by SCAR or other international bodies are recalled. The reduction of funds and fall in young people interested in scientific studies represent the main problems for the future work.
\end{abstract}

Key words Antarctica-atmospheric researches

\section{Introduction}

In 1985, the Italian Parliament passed a bill authorising our country to develop a research program in Antarctica, to acquire the right to enter into the Antarctic Treaty (L. n. 284, 1985).

The implementation of the program, named Programma Nazionale di Ricerche in Antartide (PNRA), was entrusted to the Ministry for University and Research, which divided the activity into two branches: the first, devoted to the science, was committed to the Consiglio Nazionale delle Ricerche (CNR), involving also Universities and Scientific Institutions such as the Istituto Nazionale di Geofisica e Vulcanologia (INGV) and the Osservatorio Geofisico Sperimentale (OGS). The other,

Mailing address: Dr. Michele Colacino, Istituto di Scienze dell'Atmosfera e del Clima (ISAC), CNR, Via Fosso del Cavaliere 100, 00133 Roma, Italy; e-mail: m.colacino@isac.cnr.it devoted to logistics, was committed to ENEA, an Institution concerned with alternative energies and environment. ENEA activity was focused either on the construction of the Italian Base of Terra Nova Bay in the Ross Sea region (fig. 1), or on organising the yearly scientific expedition in Antarctica.

The main decisional organism of the program has been and still is the Commissione Scientifica Nazionale (CSNA), whose primary job is to evaluate programs submitted as triennial proposals. These are forwarded to referees and evaluated upon considering the number of researchers involved, logistic needs, and funds requested. Only feasible programs in terms of the available resources are accepted and supported. Figure 2 shows the structure of the PNRA, where the role clearly appears of CSNA that gets inputs from scientific and logistic branches and promotes different research areas and international projects. The scientific fields of concern include several disciplines; programs in Geology, Biology, Astronomy, Geophysics, Oceanography, Atmospheric Physics and Chemistry, Environmental Impact, International Law, Medicine and Technology were approved and supported. Another important aspect dealt with 


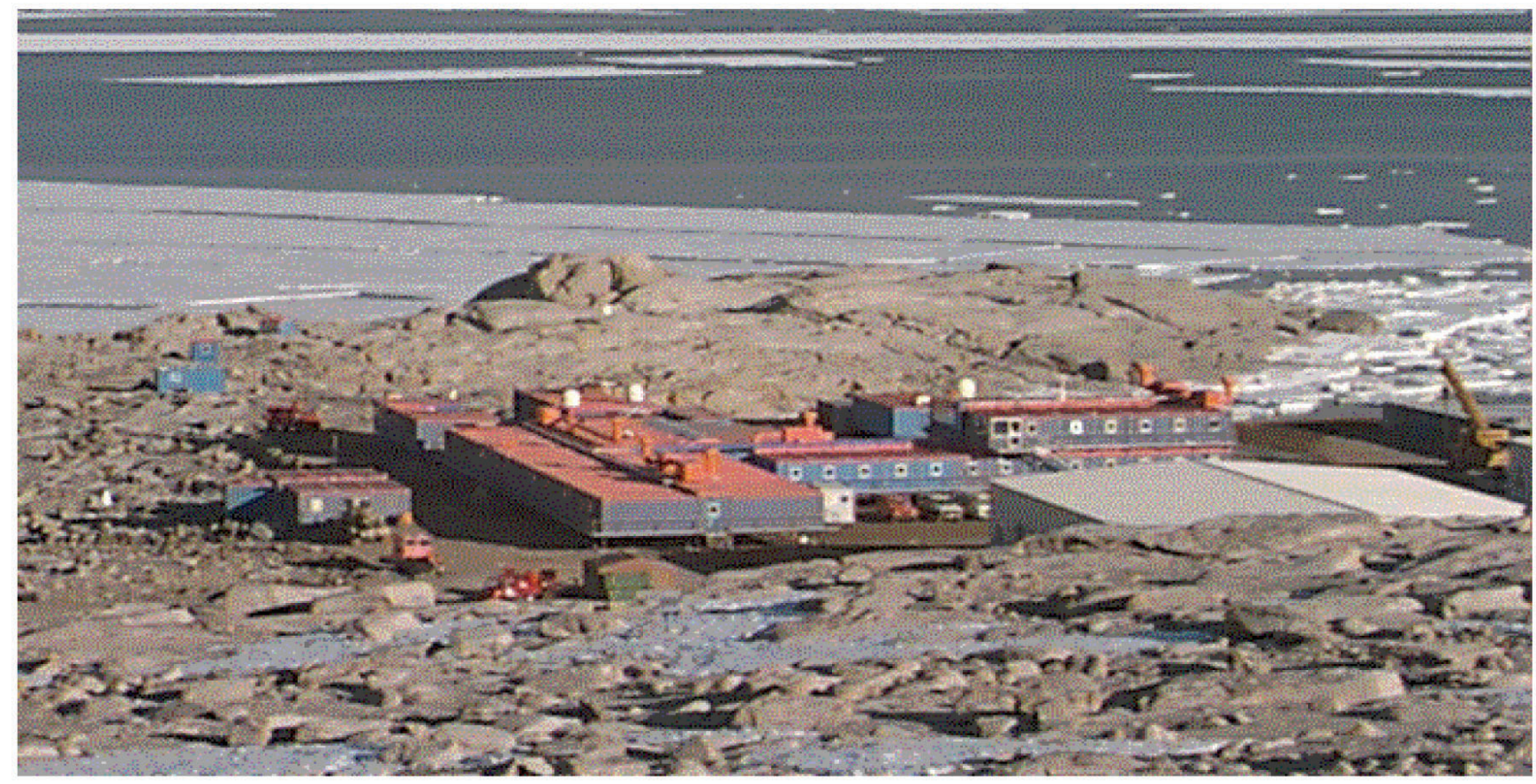

Fig. 1. A view of the Italian Base at Terra Nova Bay.

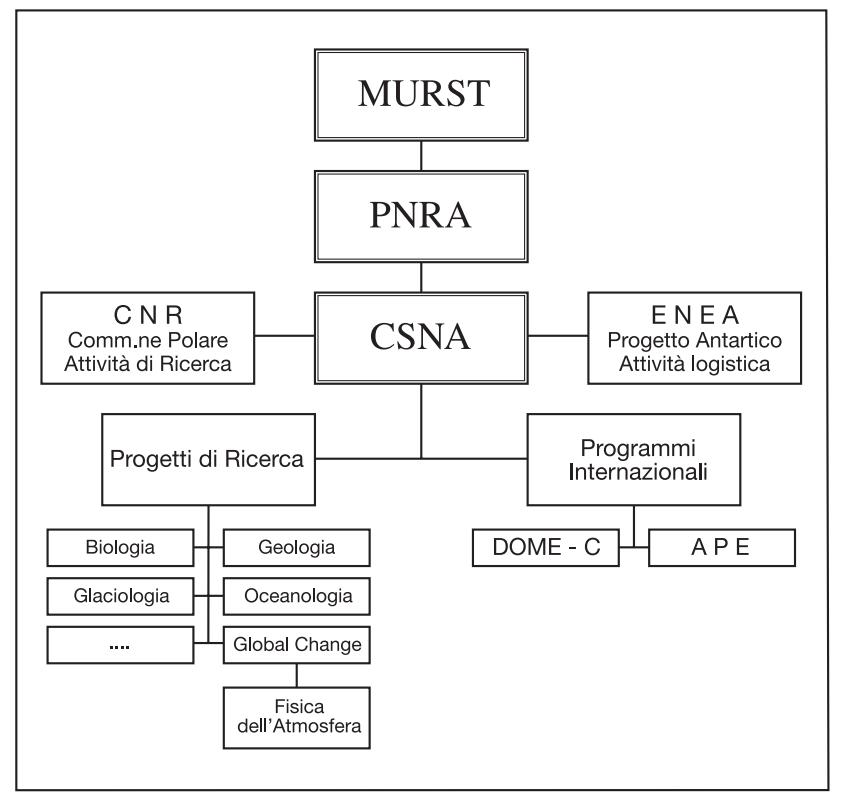

Fig. 2. Flow diagram of PNRA structure.

the international activity: in fact, several projects were launched like international co-operation, either as bilateral initiatives or as participation in programs suggested by the Scientific Committee for Antarctic Research (SCAR). Obviously, over the years several programs were concluded, others were modified, and new activities were started.

This paper presents research carried out in Atmospheric Physics, results, problems that were tackled and those waiting for a solution, while looking forward to future activities.

\section{Topics in atmospheric research}

Scientific concern aimed to establish different research lines on which to concentrate resources according to the advice of different experts, who were members of the CSNA. One of the authors (M.C.) and G. Fiocco were charged to choose activity lines for atmospheric sciences. In this choice two specific aspects were considered: the first was connected to the need for meteoclimatological data acquisition for describing the meteorology and climatology of the region, where the base is located, and for producing meteorological analyses and forecasts necessary for the base activities; the second was concerned with the need to tackle problems of polar 
atmosphere by exploiting previously acquired know-how. In this way, Italy in a short time reached a research level comparable with other countries already active in Antarctic studies.

At present, the following research lines are operative:

- Meteorology and Climatology;

- Planetary Boundary Layer Physics;

- Aerosol, Radiation and Clouds;

- Tropospheric Chemistry;

- Physics and Chemistry of the Stratosphere; and in each line some specific projects were exploited.

Concerning the first topic, the activity developed along two lines: first, a network of Automatic Weather Stations (AWS) was implemented, gathering meteorological data throughout the year (fig. 3).
Such data base, together with data provided by the European Centre for Medium Range Weather Forecast (ECMWF), is used to feed the Weather Office created in the base. In addition, the data coming from neighbouring stations are gathered and during the summer time radiosondes are launched. The stations have already been working for over 10 years and have given the possibility of effecting a first mesoscale climatological description of the area of Terra Nova Bay (Colacino et al., 2000). In addition to these operational aspects, scientific work was done to deepen our knowledge of some meteorological phenomena typical of Antarctica, such as cyclogenesis, polar low evolution, and catabatic winds. Such studies were supported by a conspicuous theoretical research based on the use of limited area models. The activity ought

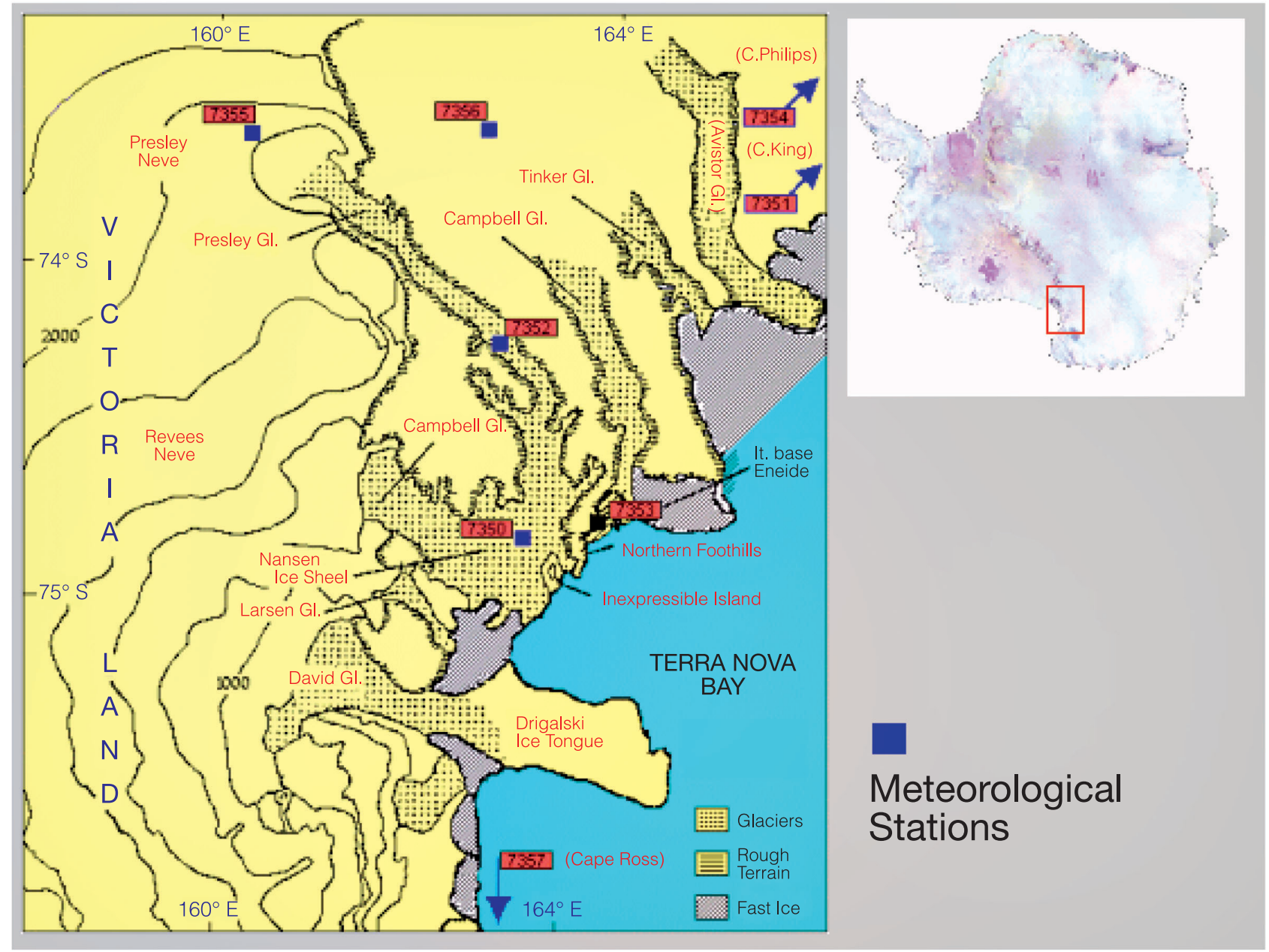

Fig. 3. The Italian mesoscale AWS network. 
be recalled done in international co-operation, as the Italian scientists contributed to the First Regional Observing Study of the Troposphere (FROST) project. It was proposed by WGPhysics and Chemistry of the Atmosphere (PACA) of SCAR and consisted in the study of Antarctic meteorology, and in determining the validity of operational analyses and forecasts relative to the more typical meteorological situations occurring both in the continent and in the surrounding oceanic areas. It was based on three special observing periods during Antarctic winter, spring, and summer, respectively. The results are included in a handbook, also available on $\mathrm{CD}$, that can be used by forecasters (Turner and Pendlebury, 2001).

More complex researches were carried out in the study of PBL. The work involved different groups that faced different problems. First of all, the study of the interaction between atmosphere and surface, both ice and water. The aim was to understand how the wind blowing from the plateau affects the energy exchange and modifies the surface energy balance, and also how the strong catabatic winds can contribute to the formation of the polynyas. Such study was carried out by means of measurement stations installed along a line, starting from the plateau to the sea and composed of poles equipped at different levels with thermometers, hygrometers, and anemometers to apply the similarity theory for the evaluation of turbulent energy exchange. In addition radiometers were used for a complete description of the radiative field. At the same time, measurements with fast answer equipments, such as sonic anemometers, were effected with the purpose of applying the theory of turbulence: in this way, a comparison of the results obtained by the two different methods was possible. By such data, it was also possible to make a description of the micrometeorology of Terra Nova Bay, useful for colleagues working on environmental impact. The studies of PBL physics, however, also include surveys carried out by means of SODAR for describing the structure of the catabatic winds. The SODAR, which is an acoustic radar, is able to give the three components of the wind vector, by analysing the acoustic signal backscattered from the atmosphere. Tri-axial equipment was installed at the Italian Base, and the structure of the wind field was analysed up to about $1000 \mathrm{~m}$. Initial campaigns were carried out in Terra Nova Bay and a statistical analysis of the wind frequencies was done using the data both of SODAR and of AWS, recorded in two subsequent summers. Such analyses indicated that the local circulation was largely dominated by catabatic winds originating by radiative cooling of near-surface air along the slopes of Reeves and Priestley glaciers. Experiments of such kind were also carried out in co-operation with French colleagues working in the base of Dumont d'Urville, that lies on the Antarctic coast, where strong catabatic winds blow almost all the year. The SODAR worked very well, showing that equipment of such kind may play an important role for studying the PBL in remote regions where other profiling techniques (kitoons, slow ascent balloons) are difficult and expensive. As a conclusion to the first cycle of measurements, the Italian group was requested either to participate in the International Project «Catabatisme et Climat» or to install SODAR equipment in the new base of Dome-C. It ought to be recalled that in the frame of the project ARTIST, supported by the European Union, similar measurements were exploited in the Arctic base of Ny-Ålesund (Svalbard Islands), for a comparison between phenomena recorded in different polar areas.

The research carried out under the title of Aerosol, Radiation and Cloud focused on the study of the radiation field, by taking into account all the radiative components. Therefore, measurements of solar short wave, surface albedo, and long wave atmospheric radiation, were collected to evaluate a radiative balance. Much attention was paid in such studies to the aerosol amount detected by different equipments because its presence modifies the surface albedo and the entire radiative budget. In addition, the influence of tropospheric clouds on the radiation field was investigated: during about three years data relative to cloudiness were collected. A specific research was carried out in the frame of the international project ECLIPS (Experimental Cloud Lidar Pilot Study), using a LIDAR installed in the French base at Dumont d'Urville. The data obtained are relative to cloud height, thickness, and optical parameters, such 
as extinction coefficient and depolarisation ratio. All these measurements contributed to a better evaluation of the radiation field and gave the possibility of improving observations from satellite. Another important activity was concerned with monitoring the UV solar radiation. It is well known that the amount of this component of the solar spectrum that reaches the ground increases during the period corresponding to the ozone hole; actually, the measurements carried out during the period from September to October clearly show the variation related to ozone depletion. Such data are also of interest for biologists, who analyse the impact of UV radiation on living species in Antarctica.

The studies of Tropospheric Chemistry were aimed at monitoring the minor atmospheric components, paying particular attention to greenhouse gases. A station was implemented in the Argentine base of Jubany for such purpose, where $\mathrm{CO}_{2}$ concentration is recorded continuously. Data collected are sent to the World Centre: the measurements in fact are done according the standards of the World Meteorological Organisation (WMO). Other measurements were collected for evaluating the concentration of $\mathrm{CFC}, \mathrm{NO}_{x}$ and other pollutants. Such samplings were collected because the Antarctica can be assumed as a reference area where the anthropogenic emissions are either absent or strongly reduced; therefore the concentration of pollutants recorded is indicative of the impact of the anthropogenic activities on the global scale. In this overview, the work developed by means of some spectrophotometers to monitorize the vertical-column content of Ozone, Sulphur Dioxide, and other trace gases should also be recalled. Observations were effected both with Brewer and Gascode equipment, not only in the Italian Base, but also at the bases of Scott (New Zealand) and Belgrano (Argentine). It was possible to record the ozone content and evidence the decrease of concentration occurring during the period from September to October.

Concerning the last research line, the activity was very intense for two reasons: first of all in 1985 the paper of Farman et al. (1985) was published, stressing the existence of the ozone hole. In addition, in Italy a conspicuous community of researchers expert in the study of the stratosphere by LIDAR was available. As a consequence, several initiatives started for both experiments and theory analysing the phenomenon of ozone depletion. Actually, monitoring of stratospheric aerosols and clouds was carried out by three different groups: the first worked in co-operation with American colleagues and installed a LIDAR station at South Pole Base (U.S.A.); the second working with another American group, monitored the PSC in Mc Murdo, making at the same time launches of stratospheric sounding balloons, equipped with CCD camera in order to collect data on the morphology of ice crystals that compose the clouds. The importance of such clouds relies on the fact that they seem to catalyse the reactions responsible for ozone destruction. The third group worked in co-operation with French researchers in Dumont d'Urville to monitor the stratospheric aerosol and PSC and to set up a LIDAR-DIAL capable of recording vertical ozone profile. In addition, the possibility was considered of arranging a LIDAR Observatory at Dome-C, as a station of the Network for Detection of Stratospheric Changes (NDSC). In the North Pole, LIDAR equipment installed in Sodankyla (Finland) and Thule (Greenland), gathered data on the stratospheric aerosol and PSC giving the possibility of a comparison between the two hemispheres. A major contribution to the research on the stratosphere was also given by the flights of the Geophysica aircraft, which will be described in more detail in the next paragraph, devoted to international activities. All such experimental activity was further supported by the development of radiative, chemical and photochemical models.

To conclude of this short review of the scientific work developed on the Antarctic atmosphere, we recall that at present the afore mentioned research lines include 14 programs with 36 groups belonging to different bodies (Universities, National Research Council, ENEA) for a total of over 120 scientists and technicians.

\section{International activities}

As already stressed above, several researches were carried out within an international frame. 
Before describing the international programs in which our groups were involved, all bilateral cooperation ought to be briefly mentioned, with Argentine, France, Japan, New Zealand, and U.S.A., respectively.

With Argentine, the common work produced the setting up of a station for $\mathrm{CO}_{2}$ monitoring in Jubany, the installation of a Brewer spectrophotometer for monitoring the vertical content of $\mathrm{O}_{3}$ at the Belgrano base, and the availability of the Usuhaia airport for radiation measurements and Geophysica flights.

The collaboration with France was concerned both with studies of PBL physics by SODAR and with monitoring tropospheric and stratospheric clouds by LIDAR at Dumont d'Urville. In addition, the implementation of a LIDAR-DIAL and common research facilities on Atmospheric Physics and Chemistry are foreseen at the base of Dome-C.

With Japan, a research group worked for the exploitation of an aureolameter that is equipment suitable to measure the amount of the aerosol in the atmosphere.

The co-operation with New Zealand dates back several years: in fact, the New Zealand Antarctic Division supported the first Antarctic expeditions carried out by Italian scientists during the seventies. They were the starting point for subsequent PNRA development. Since the beginning of PNRA in 1985, co-operation has focused on measurement of vertical content of $\mathrm{O}_{3}$ by a Brewer radiometer in Scott Base, and on the exchange of meteorological information.

Finally, the exchange of meteorological information and the stratospheric monitoring of PSC and aerosol at Mc Murdo and South Pole bases are the topics on which the co-operation with U.S.A. was active.

International links are also concerned with other important projects, and among them the Airborne Polar Experiment (APE) and the building of the plateau base at Dome-C.

The APE project was born as a co-operation between Italy and Russia, and consisted in the use of the aircraft Geophysica (fig. 4) for collecting data while flying at heights of about $20 \mathrm{~km}$.

The aircraft was a Russian military plane M-55 converted into a research plane for investigating the lower stratosphere. The M-55 Geophysica is an all-weather single seat stratospheric aircraft, operating with all climatic conditions, day and night, with an autonomy of about $5 \mathrm{~h}$; it flies at a speed of $700 \div 750 \mathrm{~km} / \mathrm{h}$, and covers a range of about $3500 \mathrm{~km}$. It can bring a payload of $1500 \mathrm{~kg}$. It was adapted to the missions and equipped with several instruments, such as chemical and microphysical sensors or remote sounders, in order to make measurements in situ of aerosol, PSC, and chemical components. The APE project, since the Geophysica aircraft is the only one in Europe capable of flight at stratospheric height, has become a project of European interest. Several other research groups from Switzerland, Germany, France, and U.S.A. requested the possibility of installing their instruments on board. Therefore, together with the PNRA, the APE project received support by the European Union and was sponsored by the European Science Foundation (ESF). The first activity was carried out by a survey during winter 1996/1997 flying over the North Pole, taking off from Rowaniemi in Finland. It was later pursued by a survey at tropical latitudes, in spring 1998. Finally in September and October 1999, the Antarctic campaign was exploited flying from Usuhaia in the Fire Land to the Antarctica peninsula. All the campaigns were aimed to collect data on the physical parameters and chemical components in the stratosphere: the North Pole measurements were carried out taking off from a base lying on the Polar circle and gave the possibility of studying the polar chemistry in the absence of a stable polar vortex. The tropical campaign was carried out to study the convective phenomena and analyse the exchange between tropospheric and stratospheric air masses. The South Pole campaign was effected from a base lying at lower latitudes $\left(55^{\circ} \mathrm{S}\right)$ giving the possibility to analyse the stratospheric chemistry trough the rim of polar vortex. All flights were successful, and during the period of the survey other research groups carried out several other related experiments.

Concerning the base at Dome-C (fig. 5), the project started with the aim to establish on the Antarctic plateau a station for NDSC. The base, whose construction is in progress, lies on the Antarctic continent at a height of $3230 \mathrm{~m}$ a.s.l. and at $1200 \mathrm{~km}$ from Terra Nova Bay and 1100 


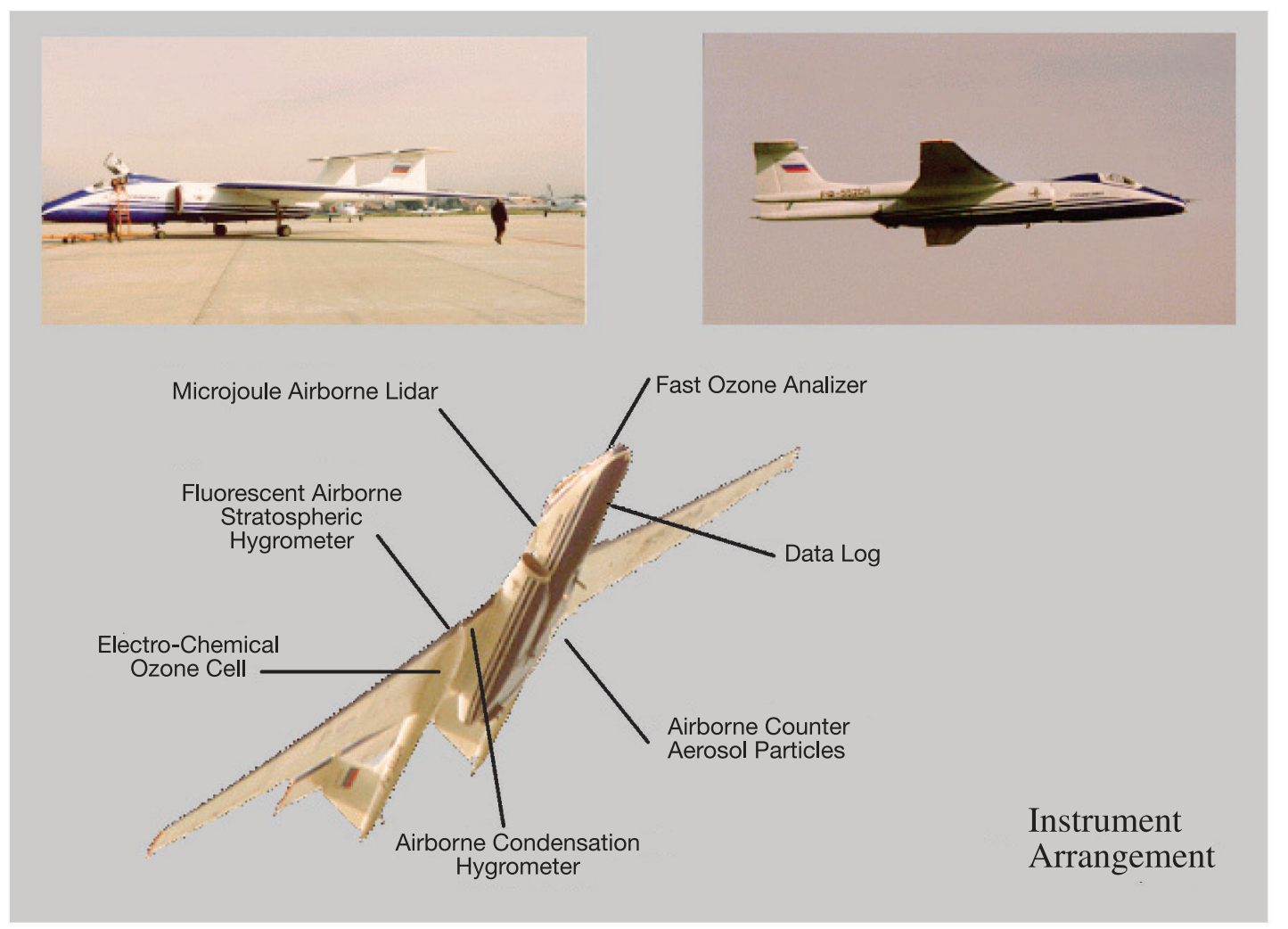

Fig. 4. The M-55 Geophysica aircraft.

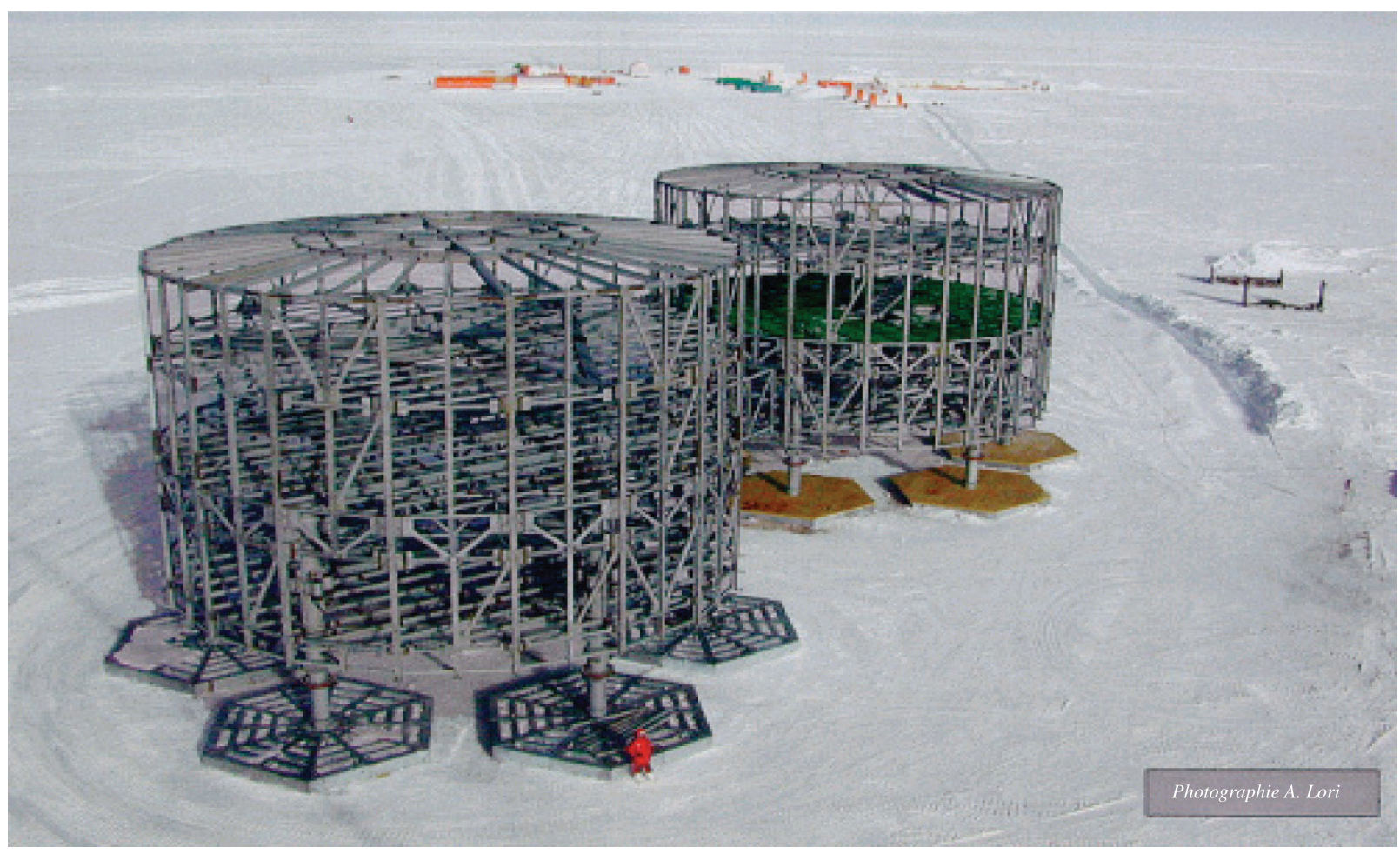

Fig. 5. The Continental Base Dome-C (work in progress). 
$\mathrm{km}$ from Dumont d'Urville. At the beginning the planned activity concerned the study of the stratosphere; as the building of the base progressed the spectrum of possible activities was expanded upon considering not only atmospheric studies, but also other matters such as glaciology, geology, biology, and astronomy. At present, the atmospheric research foreseen for the future at the base includes meteorology, PBL, and atmospheric chemistry in addition to those concerning the stratosphere.

It would be beyond the frame of the present note to give details about the results obtained and the objectives reached during 15 years of activity. We only recall that papers published in the different lines numbered over 500, more than half of which appeared in refereed Journals (PNRA pubblications, 1998). In addition, we adopted the method of meeting every two years with

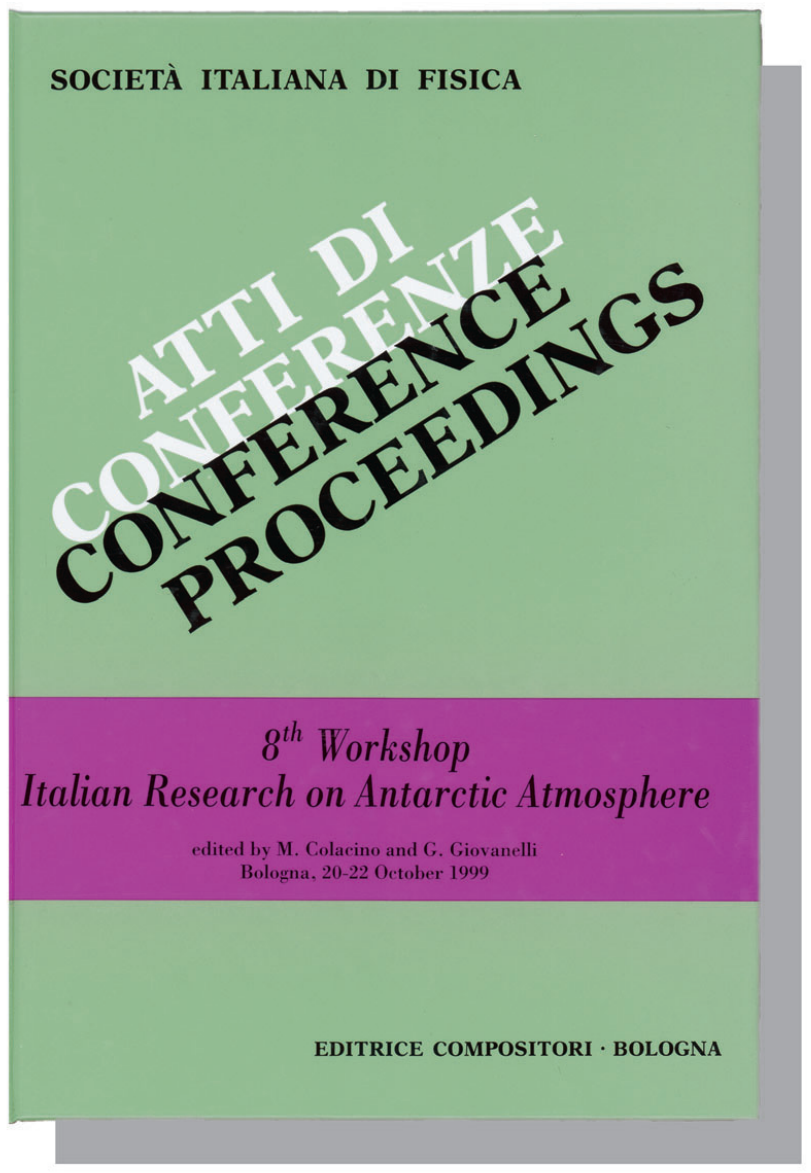

Fig. 6. Cover of the last volume of Proceedings of Workshop on Antarctic Atmosphere Research (Colacino and Giovanelli, 2000). the aim of presenting the results, discussing the problems, making proposals for new initiatives. Proceedings of the workshops (fig.6) represent the results of our meetings and the series of these books includes eight volumes. The last meeting was held in Rome in October 2001.

\section{Future perspective}

Looking to the future, the foreseen activities can be divided in two groups: i) activities of monitoring and ii) new research programs.

Concerning point (i), first of all we recall the project Reference Antarctic Data for Environmental Research (READER) suggested at the XXV SCAR meeting by the WG-PACA, and confirmed during the Tokyo meeting. It concerns the creation of a unique data set of Antarctic climatological data to be used in research. This need had already arisen during the development of the FROST program, and the availability of a certified data set seems at present of great interest due to the variability and variations in Antarctic climate. In addition, these data can also be used for studies of biology, glaciology and environmental impact. The contribution of Italy can be represented by the continuation of meteorological data collection within the AWS network; the continuous monitoring of $\mathrm{CO}_{2}, \mathrm{NO}_{x}$, $\mathrm{O}_{3}$ and of other minor atmospheric constituents. Another topic concerns the setting up of a network of solar multispectral radiometers for recording solar radiation and determining the radiative properties of aerosol. Particular attention will be paid to the UV component, due to its biological impact. Finally, the presence of Italian stations in the NDSC should be strengthened, and new observation points at Dome-C and Usuhaia could be set up.

Concerning point (ii), another initiative should be recalled. The Polar Centre of Ohio State University (U.S.A.) proposes the project Ross Island Meteorological Experiment (RIME) aimed at better understanding the meteorology of the Ross sea region. In fact, the Ross sea sector is assumed to be representative of the processes of energy exchange over the entire continent. Therefore, an understanding of the meteorological trend of this critical area can 
shed more light on the role of Antarctica in the global climate system. The proposal foresees an experimental survey carried out in a study area, and in addition the development of some numerical mesoscale models for describing and simulating local meteorology. In this project, several Italian groups could enter with their activities: from the PBL physics to radiation measurements, from mesoscale models to satellite observations.

However, the RIME project is not the only perspective for our research. A major role will be played by the availability of the new base on the continent. At Dome-C studies are foreseen in co-operation with French colleagues on meteorological and climatological phenomenology, which include both experimental and theoretical approaches. In addition, studies on the tropospheric chemistry are proposed: last April a Workshop was held in Rome devoted to a first review of such an initiative (Colacino, 2001).

It is beyond the limits of the present note to describe in detail the proposals submitted to CSNA. We can only say that at present we are facing two organising problems: the first is funds reduction, which obviously represents a limitation for further development of atmospheric research in Antarctica. The second, not less important, is the fall in young peoples involved in scientific studies, which also affects the entire geophysical and environmental sector and sets serious problems in foreseeing a turn-over of people working in Antarctica.

\section{REFERENCES}

Colacino, M. (Editor) (2001): Workshop on «Atmospheric Sciences in Dome C», Polar Atmospheres Series (PAS) n. 2/2001, pp.94.

Colacino, M. and G. Giovanelli (Editors) (2000): 8th Workshop on "Italian Research on Antarctic Atmosphere», Bologna, 20-22 October 1999, SIF (Editrice Compositori, Bologna), pp. 490.

Colacino, M., E. Piervitali and P. Grigioni (2000): Climatic characterization of the Terra Nova Bay Region, in Ross Sea Ecology, edited by F.M. FARANDA, L. GuGLIELMO and A. IONORA (Springer-Verlag, Berlin), $15-26$.

FARMAN, J.C., B.G. GARDINER and J.D. SHANKLIN (1985): Large losses of total ozone in Antarctica reveal seasonal $\mathrm{ClO}_{\mathrm{x}} / \mathrm{NO}_{\mathrm{x}}$ interaction, Nature, 315, 207-210.

L. n. 284 (1985): Legge n. 284 del 10/06/1985 - G.U. n.145 del 21/06/1985.

PNRA pubblications (1998): List of publications produced with PNRA funds: 1985-1997 / Programma nazionale di Ricerche in Antartide, edited by ENEA, Progetto Antartide, Roma, Ant. 98/04, pp. 253.

TURner, J. and S. PENDleBury (Editors) (2001): The International Antarctic Weather Forecasting Handbook, available on CD-ROM at British Antarctic Survey, Cambridge, pp. 707. 